\title{
Manipulation of Electron Beam Propagation by Hetero-Dimensional Graphene Junctions
}

\author{
Zhengfei Wang and Feng Liu* \\ Department of Materials Science and Engineering, University of Utah, Salt Lake City, Utah 84112
}

D exterous manipulation of light beams underpins the two definitive technologies of our times of information age: telecommunication and the Internet. Information is processed and transferred through carefully controlled light as information carriers. In an analogy, manipulation of electron beams is expected to form the foundation for quantum information processing and quantum computing in the future. ${ }^{1,2}$ Light rays in geometrical optics are analogous to classical trajectories of electrons, whereas electron de Broglie waves can interfere. The close relationship between optics and electronics has been made possible due to the ballistic transport properties of high-mobility electrons in two-dimensional electron gas (2DEG) created in semiconductor heterostructures, and many optical behaviors such as focusing, collimation, and interference have been achieved in 2DEG devices. ${ }^{3-6}$

There are two main approaches for manipulating electron's propagation: one by applying an external field, typically an electric or magnetic field, to alter the flowing path of electrons; the other by utilizing the specially designed structures to guide the electron's propagation, which is widely used in photonic crystals for manipulating light. Recently, graphene has been proposed as a new candidate for manipulating electron beams, based on the "external field" approach. ${ }^{7-9}$ Cheianov et al. showed the electron focusing effect through a $p-n$ junction formed by applying opposite gate voltages on two sides of graphene. ${ }^{7}$ GarciaPomar et al. proposed an n-p-n graphene transistor to realize the valley electron beam splitting or collimation. ${ }^{8}$ Park et al. demonstrated electron beam supercollimation in graphene superlattices created by an www.acsnano.org
ABSTRACT We demonstrate theoretically a new mechanism for the energy-selective manipulation of electron beam by nanostructured heterodimensional graphene junctions (HDGJs). Beam splitting, collimation, and beamguide can all be realized by designing HDGJs of different dimensionality, size and orientation. Importantly, these different functions can be combined together by predesigned patterning of multiple HDGJ units in one graphene sheet, making it feasible for large-scale integration of quantum devices. Based on transport simulations, we further suggest an method to map out the electron beam propagation path through HDGJs by scanning probe microscopy.

KEYWORDS: graphene junction · graphene transport · electron beam manipulation - scanning probe microscopy

external periodic potential, ${ }^{9}$ which induces anisotropic group velocities of low-energy charge carriers. ${ }^{10,11}$

Here, we demonstrate a new method for manipulating electron beam in graphene by the "structural design" approach. We show that by creating nanostructured HDGJs, electron beam can be manipulated and controlled without applying external field. Beam splitting, collimation, and beam-guide can all be achieved by designing HDGJs of different dimensionality, size, and orientation. Because HDGJs can be potentially made by nanopatterning techniques ${ }^{12}$ compatible with the existing electronics technology, our approach makes it feasible for large-scale graphenebased quantum device integration. Based on transport simulations, we further suggest an method to map out the electron beam propagation path through HDGJs by scanning probe microscopy (SPM), similar to what has been done in 2DEG. ${ }^{2,13}$

\section{RESULTS AND DISCUSSION}

The basic concept of a HDGJ device is illustrated in Figure 1a for the case of electron beam splitting. It consists of a HDGJ made of 1D graphene nanoribbon (GNR) and 2D graphene sheet with zigzag
*Address correspondence to
fliu@eng.utah.edu.

Received for review January 27, 2010 and accepted March 26, 2010.

Published online April 2, 2010. $10.1021 / \mathrm{nn} 1001722$

() 2010 American Chemical Society 


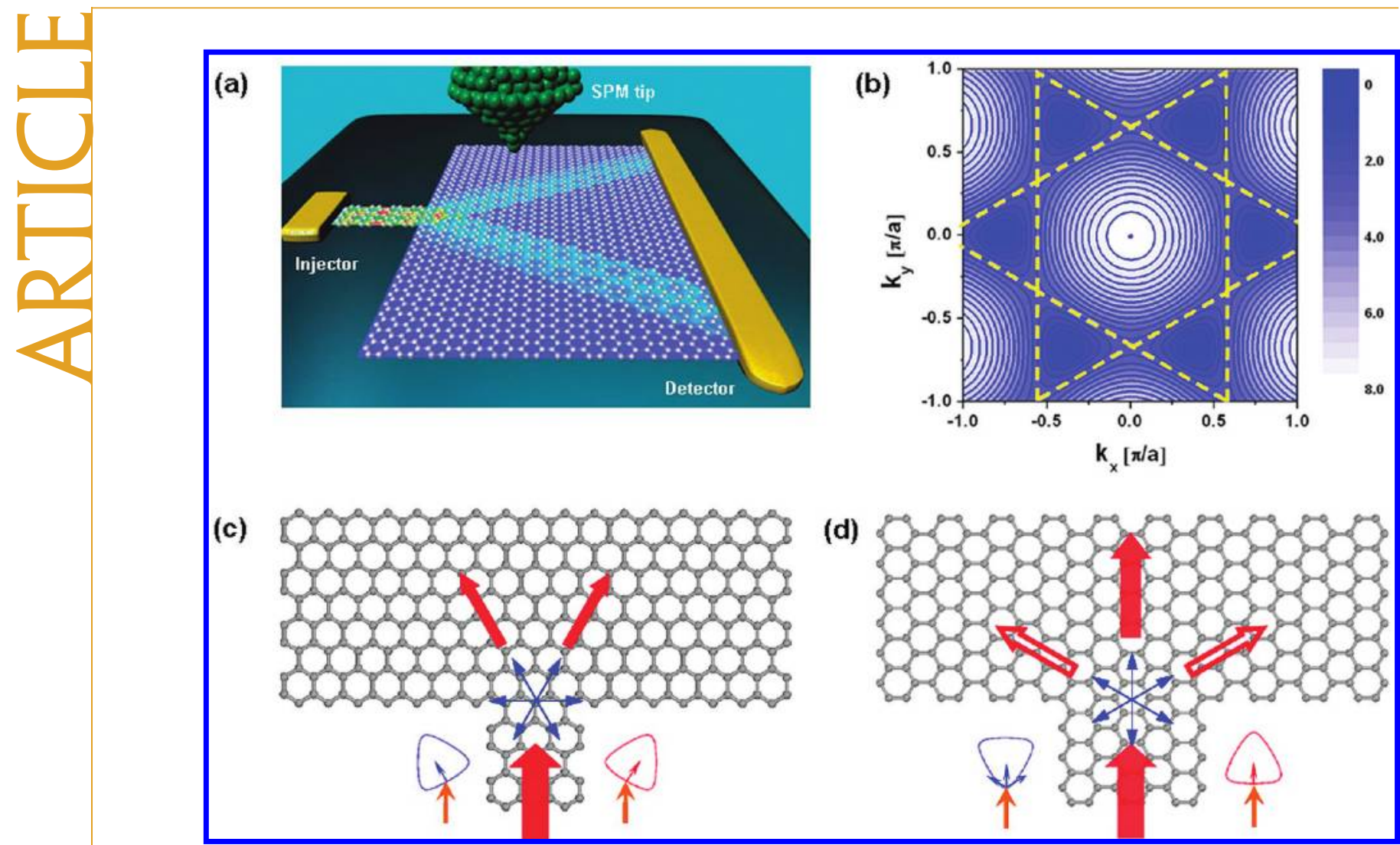

Figure 1. (a) Schematics of a 1D-2D HDGJ for electron beam splitting and SPM mapping of electron propagation paths. See detailed discussion of Figure 2 in the text. (b) Equal-energy contours of graphene band, which is hexagon at $E= \pm t$ (dashed line). 1D-2D HDGJs with zigzag (c) and armchair interface (d). Six blue arrows show the directions of anisotropic group velocity in eq 2 , the thick red arrows show preferred electron propagation directions. The blue and red equal-energy contours denote the isosurface around two nonequivalent valleys, and the thin arrows denote the electron propagation directions in different valleys. For the zigzag interface, the injected beam splits into two beams, one from each valley of different directions. For the armchair interface, the injected beam splits into three beams. The center forward beam has contributions from both valleys, and the two side beams come from one valley (blue valley on the left).

interface (i.e., the 2D graphene sheet has a zigzag edge along the interface). As an electron beam is injected into the 1D GNR, it splits into two beams at $60^{\circ}$ angle in the $2 \mathrm{D}$ graphene sheet upon passing through the $1 D-2 D$ junction. The electron beam splitting (or complete electron propagation path in general) is mapped out by measuring the conductance of the HDGJs between the injector and detector while scanning the graphene at the same time using a SPM.

Let us first discuss the physical origin of the energydependent anisotropic propagation of charge carriers in graphene. Graphene, a single layer of carbon atoms in a honeycomb structure, has recently become an active research area in nanoscience, not only because of its peculiar low-energy massless Dirac fermion band structure, ${ }^{14,15}$ but also because it holds promise for novel electronics applications. ${ }^{16-18}$ Using the nearestneighbor (NN), tight-binding (TB) model, its dispersion relation can be written as ${ }^{19}$

$$
\begin{aligned}
E_{ \pm} & = \\
& \pm t \sqrt{1+4 \cos \left(\frac{\sqrt{3}}{2} a k_{x}\right) \cos \left(\frac{3}{2} a k_{y}\right)+4 \cos ^{2}\left(\frac{\sqrt{3}}{2} a k_{x}\right)}
\end{aligned}
$$

$a=1.42 \AA$ is the carbon-carbon bond length, $t=2.66$ $\mathrm{eV}$ is the NN hopping parameter. The equal-energy contours of electron bands for $\left|E_{+}\left(k_{x}, k_{y}\right)\right|=\left|E_{-}\left(k_{x}, k_{y}\right)\right|$ are plotted in Figure $1 \mathrm{~b}$. One notices that, at low and high energies, the contours are circular, indicating isotropic electron energies in the $\mathrm{k}$-space. In contrast, the contour becomes a hexagon at $E= \pm t$, indicating highly anisotropic electron energies. This in turn suggests that the electron group velocity, normal to the equal-energy contour, is highly anisotropic at $E= \pm t$, having six preferred directions, as shown by the blue arrows in Figure $1 \mathrm{c}$ or d. At $E$ $=t$, the group velocity can be calculated $\mathrm{as}^{20}$

$$
v=\left.\frac{1}{\hbar} \nabla_{k} E\right|_{E=t}=\left\{\begin{array}{c} 
\pm \frac{\sqrt{3} a t}{\hbar}(1,0) \cos \left(\frac{3}{2} a k_{y}\right) \\
-\frac{\sqrt{3} a t}{\hbar}\left(\frac{1}{2}, \pm \frac{\sqrt{3}}{2}\right) \sin \left(\sqrt{3} a k_{x}\right)
\end{array}\right.
$$

At $E=-t$, the group velocity has the same expression as eq 2 in the reverse directions, but they are filled states in the undoped graphene, which are not to be used. This anisotropic group velocity is the intrinsic property of graphene, independent of the calculation method (see Figure S2 in Supporting Information). 


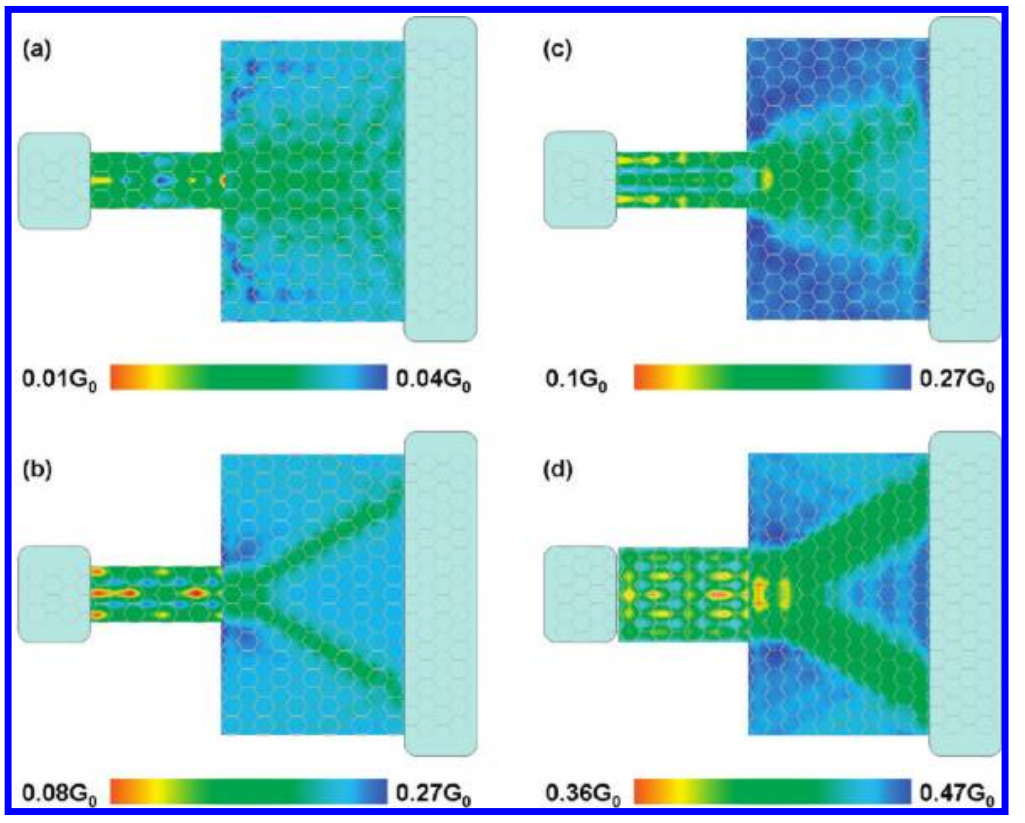

Figure 2. Zigzag 1D-2D HDGJ for electron beam splitting. The color coding shows the conductance map vs the tip position. The shaded region is the injector and detector electrode, $G_{0}=2 e^{2} / h$ is the unit of conductance. (a) $E=1.0 \mathrm{eV}$, (b) $E=2.659$ $\mathrm{eV}$, (c) $E=5.0 \mathrm{eV}$, (d) $E=2.659 \mathrm{eV}$.

The above analysis provides an interesting mechanism for designing HDGJs to manipulate electron beam propagation. Consider a 1D-2D junction formed between a 1D GNR and a 2D graphene sheet: there are two limiting cases for the orientation of the junction interface, that is, the zigzag and armchair interface, as shown respectively in Figure $1 \mathrm{c}$ and $\mathrm{d}$. Suppose an electron beam with $E \approx t$ is injected into a $1 \mathrm{D}$ ribbon passing through the junction into a 2D sheet, the zigzag junction will function as a beam divider, splitting the incident beam into two beams at an angle of $60^{\circ}$ along the directions of the largest group velocities, and each beam originates from different valley's contribution, as shown in Figure 1c. The armchair junction will function as a beam collimator, collimating the incident beam along one direction, the incident direction, as shown in Figure 1d. At first, one might think the armchair junction will split the incident beam into three beams of equal intensity, one along the direction of the incident beam (red arrow in Figure 1d) and the other two at an angle $60^{\circ}$ away from the incident direction (hollow red arrows in Figure 1d). But as pointed out by GarciaPomar $^{8}$ et al., the electron beam propagates through two valleys differently in such symmetry. In one valley, the beam is strongly collimated, propagating along the incident direction; while in the other valley, it splits, propagating equally along three directions, as shown in Figure 1d. Consequently, after passing through the armchair junction, the center beam has a much higher intensity than the two side beams, because the center beam has contributions from two valleys (contribution from one valley is three times larger), while the side beam has only one-third the contribution from one val- ley. It is such a difference in two valleys that gives rise to the beam collimation effect.

To observe the effects of HDGJs for electron beam splitting and collimation, we have mapped out the electron beam propagation paths through the HDGJs by simulating the conductance when graphene is scanned by SPM ${ }^{21,22}$ (see Supporting Information for the detailed description of the method). When the SPM tip scans over graphene, it creates a depletion region that backscatters electrons due to a locally increased onsite energy. This scattering effect is stronger when the tip scans over areas of high electron density (i.e., high electron flow) and negligible over areas of low electron flow. Consequently, the mapped-out conductance distribution is opposite of the original electron flow distribution, that is, the original maximum electron flow path shows up as a minimum in our simulated tipinduced conductance.

Figure 2 shows the numerical results of conductance map versus the tip position in the zigzag 1D-2D HDGJs, demonstrating the electron beam splitting effect. The electron flow through the HDGJs shows strong energy dependence. At $E=1.0 \mathrm{eV}$ (Figure 2a), electrons have no preferred path in the $2 \mathrm{D}$ graphene sheet after passing through the zigzag junction; their conductance exhibits a general drop "everywhere", as shown by a "uniform" distribution. This is consistent with the analysis of Figure $1 \mathrm{~b}$, as the electrons at this energy have an isotropic group velocity without any preferred propagating directions. When energy is increased to $2.659 \mathrm{eV}$ (Figure 2b), the conductance map is drastically different. The incident electron beam splits and flows predominantly along two paths at an angle of having the largest localized conductance drop. This is again consistent with the analysis of Figure 1c, showing the largest group velocity anisotropies at $E=2.66$ $\mathrm{eV}$ and the beam splitting effect of a zigzag junction. Further increasing energy to $E=5.0 \mathrm{eV}$ (Figure 2c), the group velocity become isotropic again and the beam splitting effect ceases having a conductance map similar to the low-energy case of Figure 2a. In addition, we examined the dependence of the 1D-2D HDGJ beamsplitting effect on $1 \mathrm{D}$ injector ribbon width at $E=2.659$ eV (Figure 2d). The wider injector ribbon results in wider electron propagation paths in $2 \mathrm{D}$ sheet. This is because the wider ribbon provides more energy channels for electrons to be injected into the graphene. 


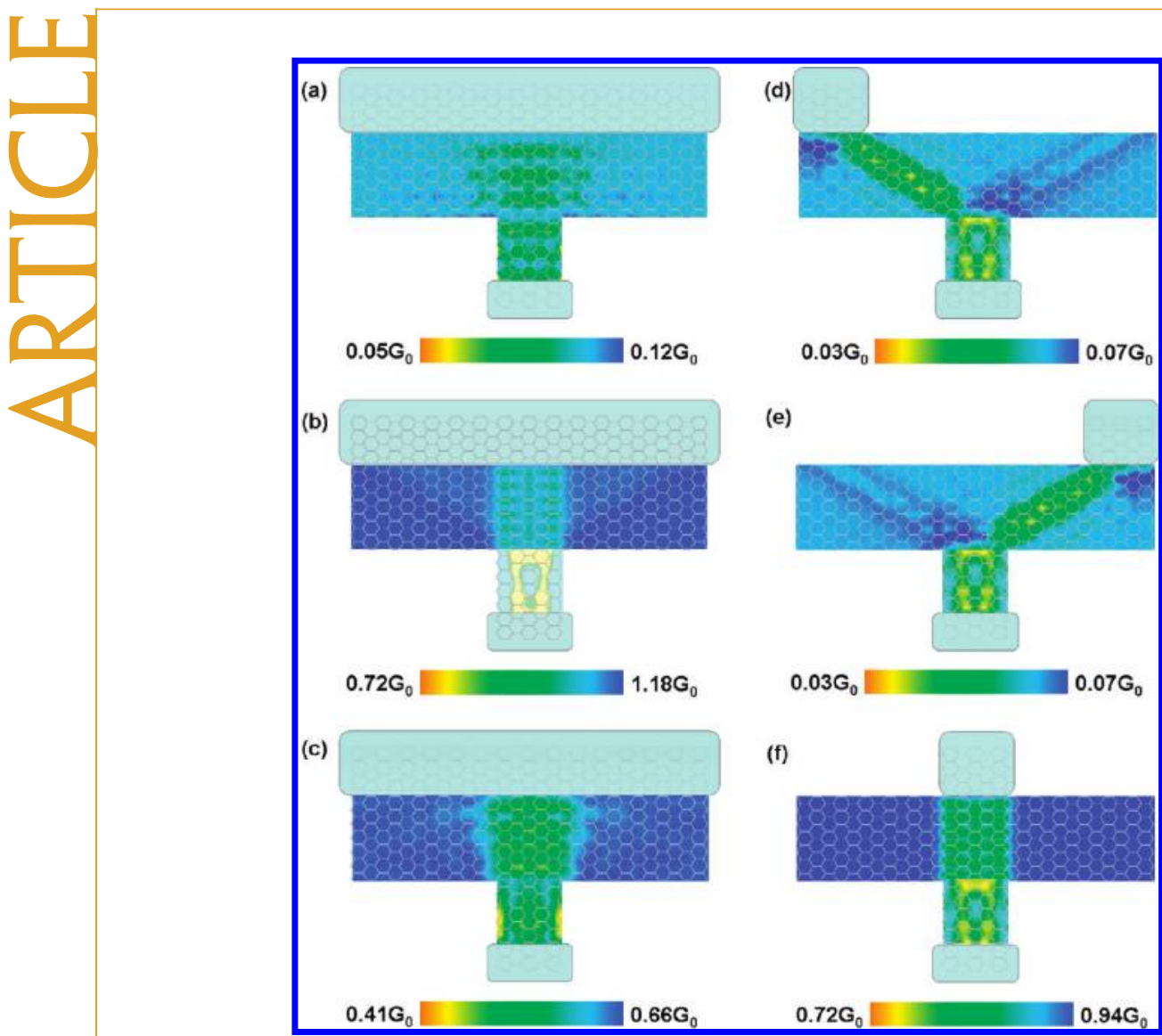

Figure 3. Armchair 1D-2D HDGJ for electron beam collimation. (a) $E=1.0 \mathrm{eV}$, (b) $E=2.659 \mathrm{eV}$, (c) $E=5.0 \mathrm{eV}$. (d), (e) and (f) $E=2.659 \mathrm{eV}$ with three local narrow detectors.

Figure 3 shows the conductance map versus the tip position in the armchair 1D-2D HDGJs, demonstrating the energy-dependent electron beam collimation effect. Consistent with the analysis of Figure 1d, after passing through the armchair junction, electrons show mainly one path along the incident direction at all energies (Figure $3 \mathrm{a}-\mathrm{c}$ ). However, at low $(E=1.0 \mathrm{eV}$, Figure $3 \mathrm{a})$ and high ( $E=5.0 \mathrm{eV}$, Figure $3 \mathrm{c}$ ) energies when elec-

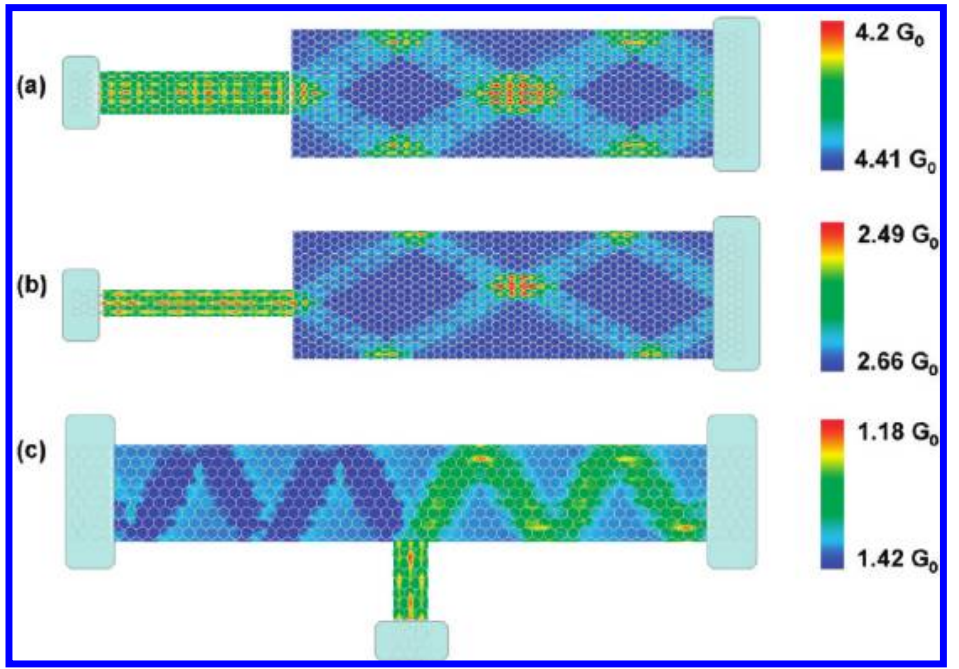

Figure 4. 1D-1D HDGJ for electron waveguide. ( $a$ and $b$ ) $E=2.659 \mathrm{eV}$ for armchair edge ribbon. (c) $E=2.659 \mathrm{eV}$ for zigzag edge ribbon, the conductance is calculated between the right detector and center injector. tron group velocities are relatively isotropic, the electron path is somewhat spread out showing rather weak collimation effect. In contrast, at $E=2.659 \mathrm{eV}$ (Figure 3b) when electron group velocity is highly anisotropic, electron path is highly collimated without spatial spreading or diffraction. As discussed above, the collimation effect of the armchair HDGJ is related to the different electron propagation in the two valleys and there exist two other much weaker electron paths away from the central beam (see Figure 1d). To reveal this phenomenon directly, we placed three local narrow detectors separately on each of the three beam pathways, as shown in Figure 3d-f. Our simulations show that all three beams can now be observed. However, the intensity of the collimated central beam (Figure $3 f$ ) is about 1 order of magnitude higher than the two split side beams (Figure 3d and e), consistent with our analysis.

In addition to electron beam splitting and collimation, it is also desirable to direct electron beam propagation directions and control beam size in quantum information processing. Next, we demonstrate such possibility by using HDGJ electron waveguide made of 1D-1D junction between two GNRs of different orientations and widths. We take advantage of the $60^{\circ}$ beam splitting effect of zigzag junctions between a narrow ribbon and a wide ribbon to realize the energyselective waveguide function, as shown in Figure 4.

When an electron beam of $E=2.659$ $\mathrm{eV}$ is injected into the narrow ribbon, it splits into two beams in the wide ribbon after passing through the zigzag junction, similar to the case of 1D-2D zigzag junctions (Figure 2). As the split beams reach the wide ribbon boundaries (edges), they will be reflected changing directions so that the wide ribbon acts as a waveguide to direct the ballistic electron propagation.

Figure 4 shows the simulated electron beam propagation in several examples of electron waveguide made of 1D-1D junction of different ribbon orientations and widths. In Figure 4a,b, the two ribbons of different widths are aligned in the same direction with armchair edges. The injected electron 
beam from the narrow ribbon is split into two beams propagating in the wide ribbon. When the two split beams reach the wide ribbon edges, they are reflected back with a $120^{\circ}$ mirror reflection, assuming their energy and momentum are conserved in an elastic scattering process with the boundary. The incoming and reflected beams cross over (overlap with) each other at the edge and the middle of ribbon, so that a low conductance region is observed. The oscillation period of the two guided beams can be tuned by the width of the wide ribbon (waveguide), while their beam width (spread) can be controlled by the width of the narrow ribbon (injector) comparing Figure 4a,b. We also simulated the electron beam with energy away from $2.66 \mathrm{eV}$, which showed no clear electron flowing path and waveguide effect, confirming the energy selectivity of the HDGJ waveguides. Figure $4 \mathrm{c}$ shows waveguide made of two ribbons orientated at $90^{\circ}$ to each other with the wide ribbon having zigzag edges. In this case, the waveguide changes the incident electron beam direction by $90^{\circ}$ propagating either along left and right, depending on the placement of detectors. In Figure $4 c$, the conductance is measured between the right detector and center injector. The flowing path of the left and right electron beam is clearly distinguished in that the right beam is characterized by a conductance drop, while the left beam is characterized by a conductance increase. This can be understood as the following. Because the conductance is measured between the right detector and the center injector, when the SPM tip scans over the right half of the waveguide, it creates a depletion region that backscatters the right-flowing electron beam so that its conductance decreases along the path. In contrast, when the SPM tip scans over the left half, it backscatters the left-flowing electron beam so that the conductance of the right-flowing beam (which is measured) increases because the total number of electrons is fixed (i.e., decreasing the left-flowing electrons effectively increases the right-flowing electrons). The situation reverses if we measure the conductance between the left detector and center injector.

In Figures 2 and 3, the 2D infinite graphene Green's fucntion is used in the simulation (see simulation method in Supporting Information), so we do not need to consider the edge effect in the 2D part of the junction. However, for the 1D-1D junction, both parts of the junction are $1 \mathrm{D}$, so their edges may change under a different environment, the on-site energy and local hopping parameter can be varied depending on the edge structure, such as $\mathrm{H}$ saturation. To check this effect, we also considered the possible edge effects by modifying the TB parameters at the edges. The hydrogenated edges can be effectively modeled by incorporating a different edge hopping parameter, and the resulting band structures of edge-modified graphene nanoribbon show very good agreement with the DFT results. ${ }^{23}$ Our simulations show that the hydrogenated

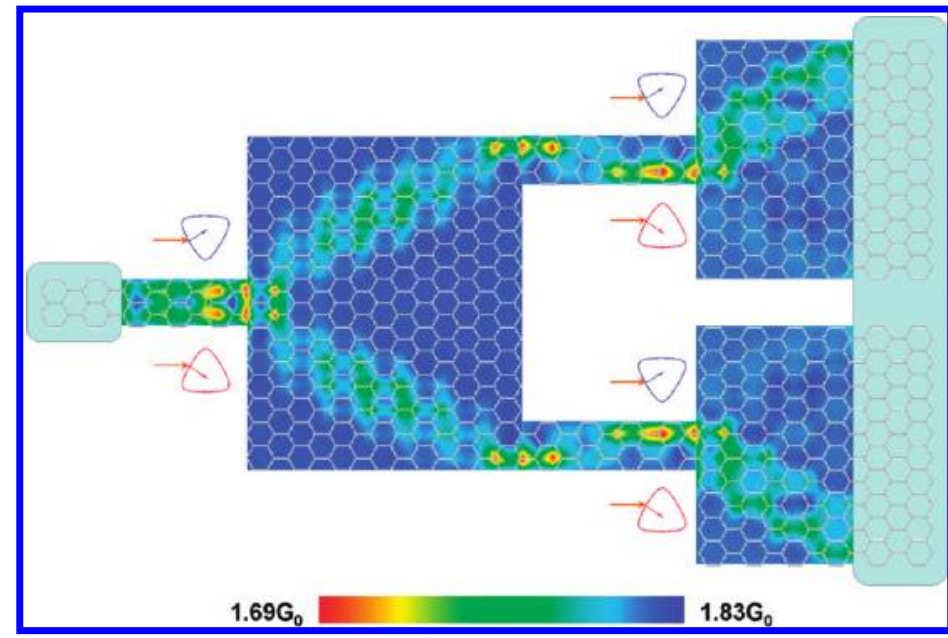

Figure 5. HDGJ integrated valleytronic device. $E=2.659 \mathrm{eV}$, and the arrows and equal-energy contours have the same meaning as those in Figure 1c.

edge has little effect on the electron flowing path comparing with the bare-edge results in our device (see Figure S3a in Supporting Information). Figures 2 and 4 show the beam splitting effect for different ribbon widths. We also simulated the effect with even wider ribbon of $\sim 3.6 \mathrm{~nm}$, which clearly shows the beam splitting effect. However, due to the computational limitation, we cannot simulate ribbon width up to what can be accessed in the real experiment $(\sim 10 \mathrm{~nm})$, but we believe our proposed device can be scaled up to function at experimentally accessible size.

One exciting potential of HDGJs to be used as electron beam manipulators is the possibility of patterning several HDGJs in one structural setting to realize novel device functionalities, such as the HDGJ valleytronic device (identifying the valley index of the splitting electron beams). In graphene, the two valleys are equivalent, so there is no intervalley scattering. However, when we consider the group velocity, which is perpendicular to the energy isosurface, the two valleys become nonequivalent. As shown in Figure $1 \mathrm{~b}$, the isosurface around the two valleys are triangular at $E=t$, so the directions of the group velocity are not the same in the two valleys. Consequently, the two valleys will have different contribution to the split beams, allowing us to identify the valley index of the split beams from their direction and intensity.

Figure 5 shows one example design of HDGJ valleytronic device. First, one incident beam splits into two beams at the 1D-1D zigzag junction, each valley contributes to only one of the splitting beams, but they are indistinguishable at this stage due to the same intensity. Next, these two beam branches are guided to flow separately along two narrow waveguides and then pass through another 1D-1D zigzag junction. At first sight, we should observe four splitting beams, two in each branch. However, the incident beams are not perpendicular to the zigzag interface so that the two splitting beams in each branch become nonequivalent at 
this stage and only one could be seen clearly, as shown in Figure 5. The high intensity beam comes from different valleys in each branch, one flow toward left and the other toward right, so that we could identify their valley indices in this process.

Our studies show that it is possible to manipulate the electron beam propagation in heterodimensional graphene junctions. We now discuss some operational limits of our proposed device. The energy of anisotropic group velocity in graphene is around the hopping energy $(t)$, so manipulating electron beam propagation requires high-energy electrons. Experimentally, this can be achieved by optical excitation ${ }^{24}$ or Schottky barrier injection of high-energy carriers. ${ }^{25}$ Also, the lifetime of these electrons in graphene is experimentally measured as $0.07-0.12 \mathrm{ps}^{24}$ consistent with the theoretical estimation of $\sim 0.05-0.1 \mathrm{ps}^{26}$ in the range of $\sim 2.5 \mathrm{eV}$, depending on carrier density. It sets up a limit on the size of the device to be $\sim 50-100 \mathrm{~nm}$ long or electrons would decay to lower energy levels if traveling beyond this distance. Recent experiments ${ }^{27-29}$ have in fact demonstrated making the HDGJs in this size range, which is really exciting.

\section{CONCLUSIONS}

In summary, our studies have demonstrated that electron beam splitting, collimation, and beam-guide can all be realized by HDGJs. The degree of electron beam spread and propagation direction can be controlled by designing HDGJs of different dimensionality, size, and orientation. One important advantage of our proposed HDGJ electron beam manipulator is that individual functions of beam splitting, collimation, and guiding can be integrated together by patterning different HDGJs in one structural setting, affording opportunities for the realization of novel device functionalities. It potentially paves the way for large-scale allgraphene device integration for quantum information processing and computing.

Acknowledgment. This work was supported by DOE CMSN and BES (Grant No. DE-FG02-03ER46027) program. We thank Center for High Performance Computing at the University of Utah for providing the computing resources.

Supporting Information Available: Conductance map simulation method and additional tests of simulation method. This material is available free of charge via the Internet at http://pubs.acs.org.

\section{REFERENCES AND NOTES}

1. Moon, C. R.; Mattos, L. S.; Foster, B. K.; Zeltzer, G.; Manoharan, H. C. Quantum Holographic Encoding in a Two-Dimensional Electron Gas. Nat. Nanotechnol. 2009, 4, 167-172.

2. Aidala, K. E.; Parrott, R. E.; Kramer, T.; Heller, E. J.; Westervelt, R. M.; Hanson, M. P.; Gossard, A. C. Imaging Magnetic Focusing of Coherent Electron Waves. Nat. Phys. 2007, 3, 464-468.

3. Houten, H. V.; Wees, B. J. V.; Mooij, J. E.; Beenakker, C. W. J.;
Williamson, J. G.; Foxon, C. T. Coherent Electron Focussing in a Two-Dimensional Electron Gas. Europhys. Lett. 1988, 5, 721.

4. Sivan, U.; Heiblum, M.; Umbach, C. P.; Shtrikman, H. Electrostatic Electron Lens in the Ballistic Regime. Phys. Rev. B 1990, 41, 7937-7940.

5. Molenkamp, L. W.; Staring, A. A. M.; Beenakker, C. W. J.; Eppenga, R.; Timmering, C. E.; Williamson, J. G. ElectronBeam Collimation with a Quantum Point Contact. Phys. Rev. B 1990, 41, 1274-1277.

6. Yacoby, A.; Heiblum, M.; Umansky, V.; Shtrikman, H.; Mahalu, D. Unexpected Periodicity in an Electronic Double Slit Interference Experiment. Phys. Rev. Lett. 1994, 73, 3149-3152.

7. Cheianov, V. V.; Fal'ko, V; Altshuler, B. L. The Focusing of Electron Flow and a Veselago Lens in Graphene p-n Junctions. Science 2007, 315, 1252-1255.

8. Garcia-Pomar, J. L.; Cortijo, A.; Nieto-Vesperinas, M. Fully Valley-Polarized Electron Beams in Graphene. Phys. Rev. Lett. 2008, 100, 236801.

9. Park, C. H.; Son, Y. W.; Yang, L.; Cohen, M. L.; Louie, S. G. Electron Beam Supercollimation in Graphene Superlattices. Nano Lett. 2008, 8, 2920-2924.

10. Park, C. H.; Yang, L.; Son, Y. W.; Cohen, M. L.; Louie, S. G. Anisotropic Behaviours of Massless Dirac Fermions in Graphene under Periodic Potentials. Nat. Phys. 2008, 4, 213-217.

11. Park, C. H.; Yang, L.; Son, Y. W.; Cohen, M. L.; Louie, S. G. New Generation of Massless Dirac Fermions in Graphene under External Periodic Potentials. Phys. Rev. Lett. 2008, 101, 126804.

12. Berger, C.; Song, Z.; Li, X.; Wu, X.; Brown, N.; Naud, C.; Mayou, D.; Li, T.; Hass, J.; Marchenkov, A. N.; Conrad, E. H.; First, P. N.; Heer, W. A. D. Electronic Confinement and Coherence in Patterned Epitaxial Graphene. Science 2006, 312, 1191-1196.

13. Topinka, M. A.; LeRoy, B. J.; Westervelt, R. M.; Shaw, S. E. J.; Fleischmann, R.; Heller, E. J.; Maranowski, K. D.; Gossard, A. C. Coherent Branched Flow in a Two-Dimensional Electron Gas. Nature 2001, 410, 183-186.

14. Wang, Z. F.; Xiang, R.; Shi, Q. W.; Yang, J.; Wang, X.; Hou, J. G.; Chen, J. Modeling STM Images in Graphene using the Effective-Mass Approximation. Phys. Rev. B 2006, 74, 125417.

15. Wang, Z. F.; Li, Q.; Su, H.; Wang, X.; Shi, Q. W.; Chen, J; Yang, J.; Hou, J. G. Electronic Structure of Bilayer Graphene: A Real-Space Green's Function Study. Phys. Rev. B 2007, 75, 085424.

16. Cresti, A.; Nemec, N.; Biel, B.; Niebler, G.; Triozon, F.; Cuniberti, G.; Roche, S. Charge Transport in Disordered Graphene-Based Low Dimensional Materials. Nano Res. 2008, 1, 361-394.

17. Wang, Z. F.; Li, Q; Shi, Q. W.; Wang, X; Yang, J.; Hou, J. G.; Chen, J. Chiral Selective Tunneling Induced Negative Differential Resistance in Zigzag Graphene Nanoribbon: A Theoretical Study. Appl. Phys. Lett. 2008, 92, 133114.

18. Wang, Z. F.; Shi, Q. W.; Chen, J. A Tunable Quantum-Dot Device Based on Cross-Bar Graphene Nanoribbon Structures. J. Nanosci. Nanotechnol. 2009, 9, 4580-4585.

19. Wallace, P. R. The Band Theory of Graphite. Phys. Rev. 1947, 71, 622-634.

20. Ueta, T. Analysis of Transport Properties of Five-Terminal Beam Splitter using Anisotropy of the Group Velocity. Electron. Commun. Jpn. 2 2002, 85, 67-75.

21. Metalidis, G.; Bruno, P. Green's Function Technique for Studying Electron Flow in Two-Dimensional Mesoscopic Samples. Phys. Rev. B 2005, 72, 235304.

22. Reynoso, A. A.; Usaj, G.; Balseiro, C. A. Magnetic Breakdown of Cyclotron Orbits in Systems with Rashba and Dresselhaus Spin-Orbit Coupling. Phys. Rev. B 2008, 78, 115312.

23. Son, Y. W.; Cohen, M. L.; Louie, S. G. Energy Gaps in Graphene Nanoribbons. Phys. Rev. Lett. 2006, 97, 216803.

24. Dawlaty, J. M.; Shivaraman, S.; Chandrashekhar, M.; Rana, 
F.; Spencer, M. G. Measurement of Ultrafast Carrier

Dynamics in Epitaxial Graphene. Appl. Phys. Lett. 2008, 92, 042116.

25. Uchida, K.; Matsuzawa, K.; Koga, J.; Takagi, S. I.; Toriumi, A. Enhancement of Hot-Electron Generation Rate in Schottky Source Metal-Oxide-Semiconductor Field-Effect

Transistors. Appl. Phys. Lett. 2000, 76, 3992-3994.

26. Tse, W. K.; Hwang, E. H.; Sarma, S. D. Ballistic Hot Electron Transport in Graphene. Appl. Phys. Lett. 2008, 93, 023128.

27. Campos, L. C.; Manfrinato, V. R.; Sanchez-Yamagishi, J. D.; Kong, J.; Jarillo-Herrero, P. Anisotropic Etching and Nanoribbon Formation in Single-Layer Graphene. Nano Lett. 2009, 9, 2600-2604.

28. Ci, L.; Xu, Z.; Wang, L.; Gao, W.; Ding, F.; Kelly, K. F.; Yakobson, B. I.; Ajayan, P. M. Controlled Nanocutting of Graphene. Nano Res. 2008, 1, 116-122.

29. Nemes-Incze, P.; Magda, G.; Kamarás, K.; Biró, L. P. Crystallographically Selective Nanopatterning of Graphene on $\mathrm{SiO}_{2}$. Nano Res. 2010, 3, 110-116. 